\title{
Knockdown of the c-Jun-N-terminal kinase expression by siRNA inhibits MCF-7 breast carcinoma cell line growth
}

\author{
EDUARDO PARRA $^{1}$ and JORGE FERREIRA ${ }^{2}$ \\ ${ }^{1}$ Biomedical Experimental Laboratory, Faculty of Sciences, University of Tarapaca, Avenida General Velásquez 1775 , \\ Arica; ${ }^{2}$ Programme of Molecular and Clinical Pharmacology, ICBM Medical Faculty, University of Chile, \\ Avenida Independencia 1027, Independencia, Santiago, Chile \\ Received May 19, 2010; Accepted July 22, 2010
}

DOI: $10.3892 /$ or_00000991

\begin{abstract}
We have examined the effect of two small interference RNA against Jnk-1 and Jnk-2 in the breast cancer cell line MCF-7. The expression of the JNK-1 and JNK-2 is frequently elevated in breast cancer and is a frequent genetic abnormality in this malignancy. For a better understanding of its role in maintaining the malignant phenotype, we used small RNA interference (siRNA) directed against Jnk-1 or Jnk-2. We made control and Jnk-1 and Jnk-2 siRNA using vector plasmid, which was then transfected to reduce its expression in MCF-7 cells. We assessed the effects of JNK-1 or JNK-2 silencing on cell growth by Western blot analysis, soft agar assay, cell proliferation assay, cell viability by MTT assay and caspase assay in vitro. Our data showed that siRNA against Jnk-1 or Jnk-2 markedly and durably reduced its expression in MCF-7 cells by up to $70 \%$, decreased the growth rate of MCF-7 cells, inhibited colony formation in soft agar and significantly reduced cell growth in MCF-7 carcinoma culture cell line. We also found that depletion of JNK-1/2 in this manner promoted apoptosis of MCF-7 cells upon serum withdrawal. In addition, we found that MCF-7 cells did not exhibit any caspase- 3 activity. In conclusion, we observed that JNK-1 and JNK-2 have a pivotal function in the development of breast cancer. Our data show that decreasing the JNK-1 or JNK-2 protein level in MCF-7 cells by siRNA could significantly inhibit MCF-7 cell growth in in vitro assay, and imply the therapeutic potential of siRNA on the treatment of breast cancer by targeting overexpression kinases such as JNK-1/2 and might be a potential therapeutic target for human breast cancer.
\end{abstract}

Correspondence to: Dr Eduardo Parra Villegas, Biomedical Experimental Laboratory, Sciences Faculty, University of Tarapaca, Avenida General Velásquez 1775, Arica, Chile

E-mail: eparra@uta.cl

Key words: c-Jun-N-terminal kinase, small interfering RNA, MCF-7

\section{Introduction}

Breast cancer is the leading cause of cancer death in women worldwide. Despite advances in detection and chemotherapy, many women with breast cancer continue to die of this malignancy $(1,2)$. Therefore, a better understanding of the nature and underlying causes of breast cancer alterations will lead to better treatment for breast cancer. c-Jun NH2-terminal kinases (JNKs), is believed to participate in most aspects of cellular function, including replication, growth, metabolism, differentiation and apoptosis $(3,4)$. JNKs, belonging to the group of stress-activated protein kinases (SAPKs), are encoded by three genes, namely Jnk-1 and Jnk-2 that are ubiquitously expressed, and Jnk-3, whose expression is restricted to the brain, heart and testis $(5,6)$. The role of JNKs in apoptosis is unclear as these proteins have been assigned both proand anti-apoptotic properties. However, there are also studies demonstrating JNK to be insignificant in regulation of apoptosis. JNK becomes activated through phosphorylation and some tumor cell lines contain constitutively active JNK (5). Anti-sense JNK oligonucleotides have been reported to inhibit the growth of such tumor cells and to induce apoptosis (7). In contrast, activated JNK is required for UVinduced apoptosis and is suggested to be essential for cytochrome c release from mitochondria in mouse fibroblasts (8). Nevertheless, the role of JNKs in several cancers has been extensively examined in many studies for the past decade (9); however, specifically reducing its level by genetic means in established breast cancer cell lines is still helpful for a better understanding of its role in maintaining the malignant phenotype. Thus, in this study, we investigated whether specifically decreasing the protein level of JNK-1/2 in a breast cancer cell line in which this protein was overexpressed might result in the inhibition of cell growth in vitro. For this purpose, small RNA interference (siRNA) directed against JNK-1/2 was used. Small interfering RNA (siRNA), sometimes known as short interfering RNA or silencing RNA, was originally identified as an endogenous mechanism for post-transcriptional gene silencing in plants and nematodes and is now an established technique for experimental 'knockdown' of gene expression to establish function in mammalian cells $(10,11)$. RNAi has typically been achieved in cultured mammalian cells by transfection of chemically synthesized genespecific 21 nt small interfering RNAs (siRNA). Introduction 
of these molecules leads to reduced expression of specific mRNAs, thus permitting the analysis of a knockdown phenotype without the need for time-consuming gene-targeting studies. The effect of siRNA involves post-transcriptional gene silencing via a process in which double stranded RNA (dsRNA) inhibits gene expression in a sequence-dependent manner through degradation of the corresponding mRNA. Its blocking action on gene expression has been successfully observed in rat and human cells cultured in vitro, and the knockdown of genes in cells has been achieved (12-15). In addition, the development of retroviral constructs in which the polymerase-III H1-RNA gene promoter synthesizes siRNA-like transcripts, allows stable expression of siRNA, thus rendering this technology applicable as gene transfer $(16,17)$. In addition, emerging evidence supports a role for JNK in stress-induced mitochondrial apoptotic pathways in a variety of cell systems (18).

Thus, the ability to stably express siRNA from a retroviral vector allowed JNK mRNA and JNK-1/2 proteins to be knocked down inducing apoptosis and loss of the adherence capacity of the breast carcinoma MCF-7 cells in soft agar (18). In the present study, siRNA targeting to the Jnk-1 and Jnk-2 genes were introduced into breast carcinoma cell lines MCF-7 which overexpresses JNK-1 and JNK-2 proteins. These observations strongly suggest that JNKs are involved in breast cancer progression despite their known role as an apoptosis inductor in several other types of human cancers $(18-20)$.

\section{Materials and methods}

Cell lines and culture. The human breast carcinoma cell line MCF-7 was a present from Dr Dan Mercola (The SKCC, La Jolla, CA, USA). The cells were cultured in RPMI-1640 medium supplemented with $100 \mathrm{ml} / \mathrm{l}$ fetal bovine serum (FBS), 8x10 $\mathrm{U} / 1$ penicillin and $0.1 \mathrm{~g} / 1$ streptomycin in humidified incubator containing $50 \mathrm{ml} / 1 \mathrm{CO}_{2}$ at $37^{\circ} \mathrm{C}(14,15)$. The antibodies used for Western blotting included those against protein kinase JNK-1 and JNK-2, Bax, Bcl-2 (Santa Cruz Biotechnology, Santa Cruz, CA, USA). Caspase-3, caspase-7, caspase-9, cytochrome c and $\beta$-actin (Sigma, St. Louis, MO, USA), cytochrome oxidase subunit IV (Cox IV; Invitrogen, Carlsbad, CA, USA), Western blot analysis was performed as previously described $(14,15)$.

siRNA preparation and transfection of small interfering $R N A$. siRNA oligonucleotides with two thymidine residues (tt) at the 3 '-end of the sequence were designed for JNK-1 (sense, 5'-AAGCCCAGTAATATAGTAGTA-3'-hairpinTTCAAGAGA-3' antisense, 5'-TACTACTATATTACTGG GCtta-3'). JNK-2 (sense 5'-CATGATGTTATCATATCT TAT-3', antisense 5'-ATAAGATATGATAACATCAtta-3'). Cells were treated in parallel with a non-silencing siRNA (sense, 5'-AATTCTCCGAACGTGTCACGT-3'; antisense, 5'-ACGTGACACGTTCGGAGAATT-3') as control oligonucleotides were synthesized by Shanghai Genechem Co. These cells were cultured in medium without antibiotics, and $24 \mathrm{~h}$ before transfection resulting in a confluence of the cell monolayer by $60-80 \%$. pTER, pTER-Jnk-1 and pTER-Jnk-2 siRNA or non-silencing siRNA $(70 \mathrm{nmol})$ were mixed with
Lipofectamine $^{\mathrm{TM}} 2000$ (Invitrogen) according to manufacturer's recommendation and added to the cells. After $6 \mathrm{~h}$ at $37^{\circ} \mathrm{C}$, the medium was changed, and the cells were cultivated in RPMI-1640 supplemented with $10 \%$ heat-inactivated FBS $(14,15)$.

Western immunoblot analysis. MCF-7 breast carcinoma cells $\left(5 \times 10^{5}\right)$ were seeded onto 6-well plates. Forty-eight hours after transfection, cells were collected and washed twice by cold PBS, and each well was treated with $50 \mathrm{ml}$ lysis buffer (2 mmol/l Tris-HCl, pH 7.4, $50 \mathrm{mmol} / \mathrm{l} \mathrm{NaCl}, 25 \mathrm{mmol} / \mathrm{l}$ EDTA, $50 \mathrm{mmol} / 1 \mathrm{NaF}, 1.5 \mathrm{mmol} / 1 \mathrm{Na}_{3} \mathrm{VO}_{4}, 1 \%$ Triton X-100, $0.1 \% \mathrm{SDS}$, supplemented with protease inhibitors $1 \mathrm{mmol} / \mathrm{l}$ phenylmethylsulfonylfluoride, $10 \mathrm{mg} / 1$ pepstatin, $10 \mathrm{mg} / 1$ aprotinin and $5 \mathrm{mg} / \mathrm{l}$ leupeptin) (all from Sigma). Protein concentrations were determined using the Bradford protein assay. Equal amounts of protein $(50 \mu \mathrm{g})$ were separated on a $15 \%$ SDS polyacrylamide gel and transferred to a nitrocellulose membrane (Hybond C, Amersham, Freiburg, Germany). Membranes were blocked in 5\% non-fat dry milk in TBS for $1 \mathrm{~h}$ at room temperature and probed with rabbit anti-JNK-1 (SC-1648) and anti-JNK-2 (SC-571) antibodies (dilution, 1:500 Santa Cruz Biotechnology) overnight at $4^{\circ} \mathrm{C}$. After 3 washes with TBS containing $0.1 \%$ Tween-20, membranes were incubated with anti-rabbit IgGhorseradish peroxidase (1:5000, Santa Cruz Biotechnology), and developed by luminal mediated chemiluminescence (Appylgen Technologies, Inc., China). To confirm equal protein loading, membranes were reproved with a 1:1000 dilution of an anti-actin antibody (Santa Cruz Biotechnology). Densitometric analyses were performed using Scion Image software.

Reverse transcription polymerase chain reaction. MCF-7 cells $\left(5 \times 10^{5}\right)$ were seeded onto 6-well plates. Total RNA was extracted $48 \mathrm{~h}$ after transfection using TRIzol reagent. Reverse transcription was performed using one step RT-PCR kit. The primers of Jnk-1 were 5'-CGT CTG GTG GAA GGA GAG AG-3' (forward primer) and 5'-TAA TAA CGG GGG TGG AGG AT-3' (reverse primer). For Jnk-2 were 5'TCT GAC GTC CTG GGC TGG AC-3' (forward primer) and 5'-GCA GCA GCC CTC AGG ATC CT-3' (reverse primer). The primers of human $\beta$-actin were 5'-TCACCAATG GGACGACAT-3' (forward primer) 5'-GAAGTCCAGGGC GACGTAG-3' (reverse primer). Thermal cycle conditions were as follows: $42^{\circ} \mathrm{C}$ for $30 \mathrm{~min}, 94^{\circ} \mathrm{C}$ for $2 \mathrm{~min}$, followed by 28 cycles of $94^{\circ} \mathrm{C}$ for $15 \mathrm{sec}, 55^{\circ} \mathrm{C}$ for $30 \mathrm{sec}, 72^{\circ} \mathrm{C}$ for $1 \mathrm{~min}$, with a final extension at $72^{\circ} \mathrm{C}$ for $10 \mathrm{~min}$. RT-PCR products were visualized by ethidium bromide-stained agarose gels and the images were scanned using a UV light.

Cell growth assay. At 2 days after transfection, MCF-7 cells transfected with indicated plasmids were harvested and replated at a density of 50 cells $/ \mathrm{mm}^{2}$ in triplicate. The total cell number was quantified every 2 days with a hematocytometer and an Olympus inverted microscope. Cell viability was assessed by using trypan blue.

DNA fragmentation ELISA. This assay measures cytoplasmic histone-bound DNA fragments (mono- and oligonucleo- 

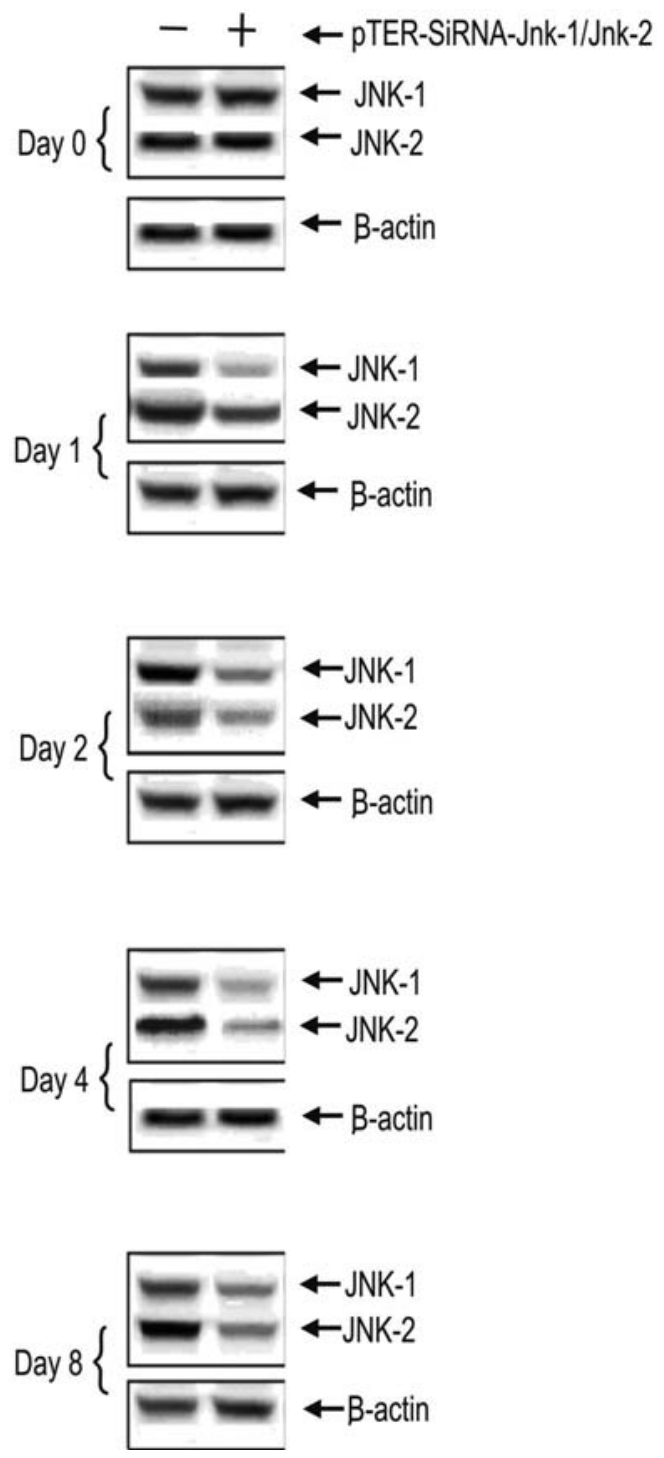

Figure 1. Time course of the reduction in JNK-1/2 protein levels by pTERJnk-1 or pTER-Jnk-2. Exponentially proliferating MCF-7 cells were transfected with, pTER, pTER-Jnk-1 or pTER-Jnk-2 and whole cell lysates were prepared at the time points indicated. Total cell lysates were separated by SDS-polyacrylamide-gel electrophoresis and immunoblotted with an antibody against JNK-1 or JNK-2; expression levels were normalized for loading by probing for $\beta$-actin. One of two similar experiments is shown.

somes) which are generated during apoptosis). The enrichment of nucleosomes in the cytoplasm of treated cells was expressed as fold induction in apoptosis compared to untreated controls. Cells $\left(1 \times 10^{5}\right)$ were incubated with $1 \mathrm{M}$ STS, washed in PBS and then lysed in lysis buffer for $30 \mathrm{~min}$. The supernatant (cytoplasmic extract) was recovered and the assay was performed according to the manufacturer's protocol (Boehringer Mannheim).

Soft agar colony formation assay. The effect of Jnk-1-siRNA or Jnk-2-siRNA on colony formation ability of the MCF-7 cells was assessed by soft agar colony formation assay. The assay was done in 6-well plates; in each well, $2 \mathrm{ml}$ of $0.5 \%$ agar (in culture medium) was layered in the bottom followed by $1 \mathrm{ml}$ of $0.38 \%$ agar as the top layer. Approximately 2000 cells were then plated over the top layer. The cells were treated with Egr-1 siRNA and maintained at $37^{\circ} \mathrm{C}$ in a humidified $5 \% \mathrm{CO}_{2}$ atmosphere. At day 14 post-treatment, plates were assessed for size and number of colonies (original magnification, $\mathrm{x} 4$ ). Colonies (in a representative field) are indicated.

Measurement of caspase activity. Cells were washed with ice-cold PBS and lysed with $100 \mu \mathrm{l}$ of lysis buffer [50 mM Tris- $\mathrm{HCl}$ (pH 7.5), $150 \mathrm{mM} \mathrm{NaCl}, 1$ mM EGTA, 1 mM NaF, $1 \%$ Nonidet P-40, $1 \mathrm{mM}$ PMSF, protease inhibitor cocktail] for $30 \mathrm{~min}$ at $4^{\circ} \mathrm{C}$. Protein extracts were collected after centrifugation at $14,000 \mathrm{rpm}$ for $10 \mathrm{~min}$. Protein concentration was determined using the Bio-Rad protein assay kit (Bio-Rad, USA). Equal amount $(50 \mu \mathrm{g})$ of protein extracts were mixed with assay buffer [20 mM HEPES ( $\mathrm{pH} 7.4)$, $100 \mathrm{mM} \mathrm{NaCl}, 0.1 \%$ CHAPS, $10 \mathrm{mM}$ DTT, 1 mM EDTA, $10 \%$ sucrose], added to 96 well microtiter plates, and incubated with $100 \mu \mathrm{M}$ of fluorogenic peptide substrates, AcDEVD-AMC (caspase-7) and Ac-LEHD-AMC (caspase-9) for $1 \mathrm{~h}$ at $37^{\circ} \mathrm{C}$. Free aminomethyl coumarin (AMC) resulting from cleavage of the aspartate-AMC bond by caspase was measured at excitation wavelength of $380 \mathrm{~nm}$ and emission wavelength of $460 \mathrm{~nm}$ using a spectrofluorometer (Geminin EM, Molecular Devices, USA).

\section{Results}

The ability to turn off individual genes at will in growing cells provides a powerful tool for elucidating the role of a particular gene and for therapeutic intervention when that gene is overexpressed or mutated. Since the discovery of siRNAs as the key mediator of RNA-induced gene silencing, RNAi has rapidly evolved to become a tool for gene regulation in mammals $(16,17)$ and have been applied to inhibit the expression of a wide variety of target genes. Two of them, the Jnk-1 and Jnk-2 genes expressing the respective kinases with important functions in the regulation of growth and differentiation, is involved in the transformed phenotype of several cancer types.

Effect of siRNA against Jnk-1 and Jnk-2 in the expression of $J N K-1$ and JNK-2 proteins in breast carcinoma cell line $M C F-7$. To examine the specific effect of siRNA treatment on JNK-1 and JNK-2 expression in MCF-7 breast carcinoma cell lines, the Jnk-1 and Jnk-2 mRNA and protein expression levels were determined quantitatively with RT-PCR and Western blot analyses, respectively (Figs. 1 and 2).

Jnk-1 and Jnk-2 mRNA were strongly expressed in MCF-7 breast carcinoma cells lines as reflected by RT-PCR (Fig. 1). The inhibition rate of Jnk-1 and Jnk-2 mRNA after transfection with specific Jnk-1 or Jnk-2 siRNA was strongly induced $24 \mathrm{~h}$ after transfection with the specific Jnk-1 or Jnk-2 siRNA. The inhibition of JNK-1 and JNK-2 protein expression in MCF-7 breast carcinoma cells was observed until day 8 after transfection (Fig. 1).

Similarly, Western blot assay of JNK-1 and JNK-2 protein expression in the MCF-7 cell line treated with pTER empty vector (-) or treated with pTER-siRNA-Jnk-1 or Jnk-2 

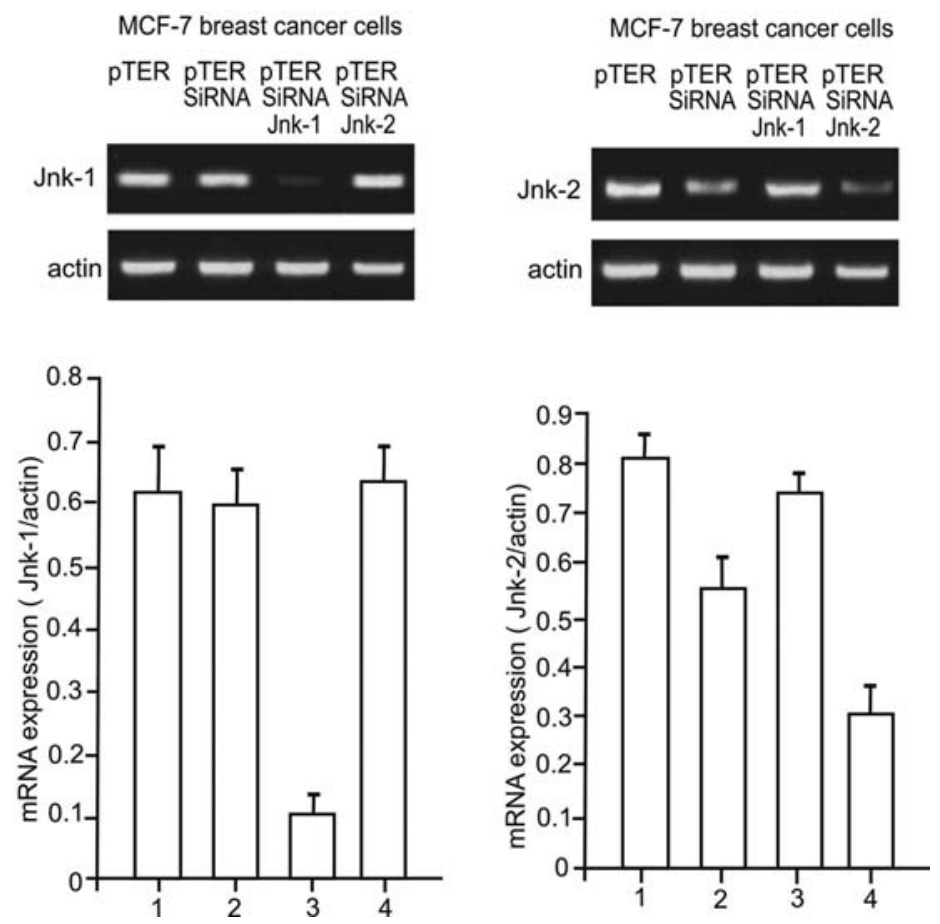

Figure 2. Expression of Jnk-1 and Jnk-2 mRNA in the breast carcinoma cell line MCF-7. Jnk-1 and Jnk-2 mRNA expression in RT-PCR assay in the MCF-7 cell line. Lane 1, treated pTER; lane 2, treated with pTER-siRNA; lane 3, treated with pTER-siRNA-Jnk-1 and lane 4, treated with pTERsiRNA-Jnk-2. Data are expressed as mean \pm SD of two experiments.

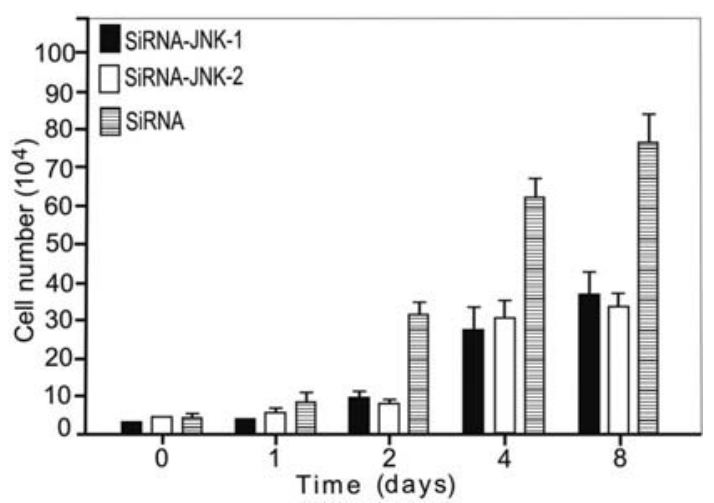

Figure 3. Knockdown of Jnk-1 or Jnk-2 by siRNA reduces colony formation in soft agar. MCF-7 cells were transfected with pTER as control, pTERsiRNA-Jnk-1 or pTER-siRNA-Jnk-2, and seeded in $0.35 \%$ agarose containing Dulbecco's modified Eagle's medium with $10 \%$ fetal bovine serum. The colony numbers were counted 2 weeks later. (A) Representative wells demonstrating the total number of colonies formed by MCF-7 transfected with the indicated plasmids. (B) The numbers of colonies of pTER, pTER-siRNA-Jnk-1 or pTER-siRNA-Jnk-2 treated cells standardized against the control cells (set at 100\%). The data shown are means \pm SD from two independent triplicate experiments.

mRNA expression plasmid (+), showed that the JNK-1 and JNK-2 protein expression in MCF-7 cells transiently transfected with the empty vector exhibited a strong JNK-1 or JNK-2 band by Western blotting (-) while, the cells treated with siRNA-Jnk-1 or Jnk-2 (+), strongly decrease the expression of JNK-1 and JNK-2 proteins (Fig. 2). B-actin protein signals were uniform in all analysed samples.

Decreased levels of JNK-1/2 significantly alter the growth rate of MCF-7 cells. Previous studies have shown that Jnk-1
A

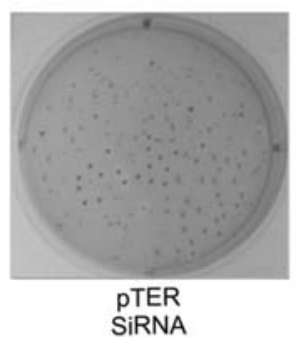

B
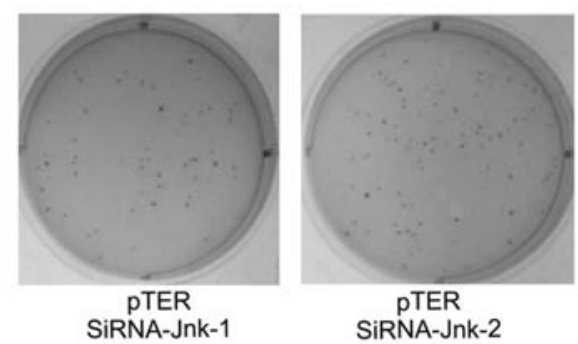

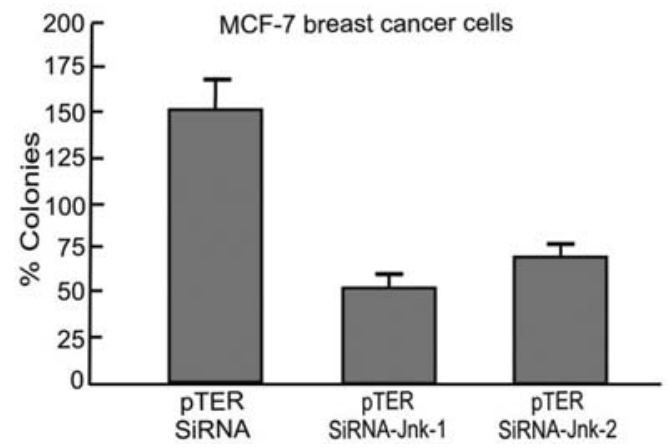

Figure 4. Protein levels of Bcl-2, Bax, following different drug exposure. MCF-7 breast carcinoma cells were treated with siRNA, siRNA-Jnk-1 or siRNA-Jnk-2. Protein $(50 \mu \mathrm{g})$ was loaded for the controls and treated samples: i) untreated cells; ii) pTER vector; iii) pTER-siRNA-Jnk-1; iv) pTER-siRNA-Jnk-2. One of two similar experiments is shown.

and Jnk-2 are important in cellular proliferation and cell growth. Thus, increased levels of Jnk-1 or Jnk-2 might have a function in the growth advantage seen in breast tumors. In 


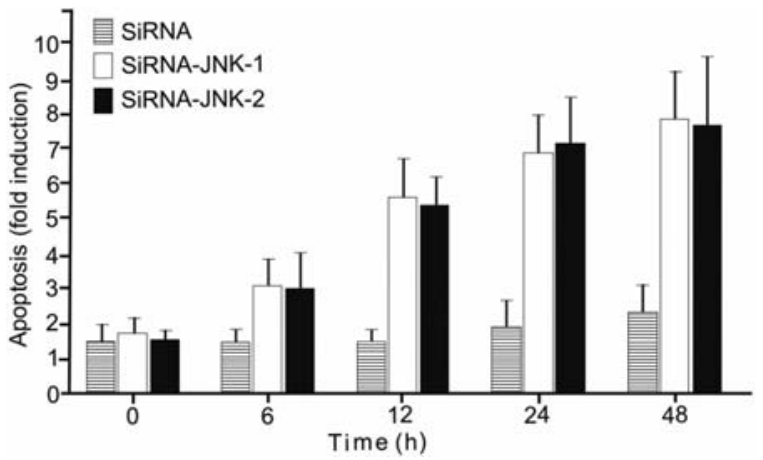

Figure 5. siRNA against Jnk-1 or Jnk-2 mRNA induced apoptosis in MCF-7 human breast cancer cells. Cells were treated with siRNA, siRNA-Jnk-1 or siRNA-Jnk-2 for the times indicated, lysates were analysed by ELISA according to the manufacturer's instructions. Fold induction in apoptosis is expressed as amount of cytoplasm DNA-histone complexes in treated cells compared to untreated controls $(0 \mathrm{~h})$. Error bars represent the mean \pm SEM of three independent experiments.

our study, MCF-7 cells were transfected with pTER, pTERJnk-1 or Jnk-2. The number of MCF-7 cells was then counted every 2 days after transfection. Our data showed that siRNA directed against Jnk-1 or Jnk-2 significantly decreased the growth rate of MCF-7 cells by $60-70 \% 48 \mathrm{~h}$ after the transfection (Fig. 3).

Decreases in JNK-1 and JNK-2 protein inhibit colony formation. We then tested whether siRNA-mediated reductions in JNK-1 or JNK-2 protein levels could influence the ability of MCF-7 cells to form colonies in soft agar. MCF-7 cells were transfected with pTER, pTER-Jnk-1 or pTER-Jnk-2. At $48 \mathrm{~h}$ after transfection, the cells were placed into medium with soft agar, and colonies were counted after 2 weeks. siRNAi directed against Jnk-1 or Jnk-2 resulted in a significant decrease (about 65\%) in colony formation in MCF-7 cells (Fig. 4). The smaller number of colonies in the pTER-siRNA-Jnk-1 or siRNA-Jnk-2 groups than in the control group were statistically significant $(\mathrm{P}<0.001)$. These results showed that the reduction in JNK-1 or JNK-2 protein level decreased the ability of breast cancer cells to form colonies in soft agar (Fig. 4).

siRNA-Jnk-1 and siRNA-Jnk-2 induce apoptosis in MCF-7 human breast cancer cells. MCF-7 cells were treated with the siRNA against Jnk-1 or Jnk-2 for the times indicated, lysates were analysed by ELISA according to the manufacturer's instructions. Fold induction in apoptosis is expressed as amount of cytoplasm DNA-histone complexes in treated cells compared to untreated controls. We examined DNA fragmentation by cell death ELISA. Results presented in Fig. 5, show a time-dependent increase in the presence of siRNA-Jnk-1 or siRNA-Jnk-2 in MCF-7 cells. The cells exhibit great levels of apoptosis after 48-h transfection. The apoptotic effect was confirmed by nuclear morphology (data not shown) (Fig. 5).

The apoptotic effect of siRNA against Jnk-1 and Jnk-2 mRNA in MCF-7 cells is not regulated by caspase-3. Emerging evidence supports a role for JNK in stress-induced mito-
A
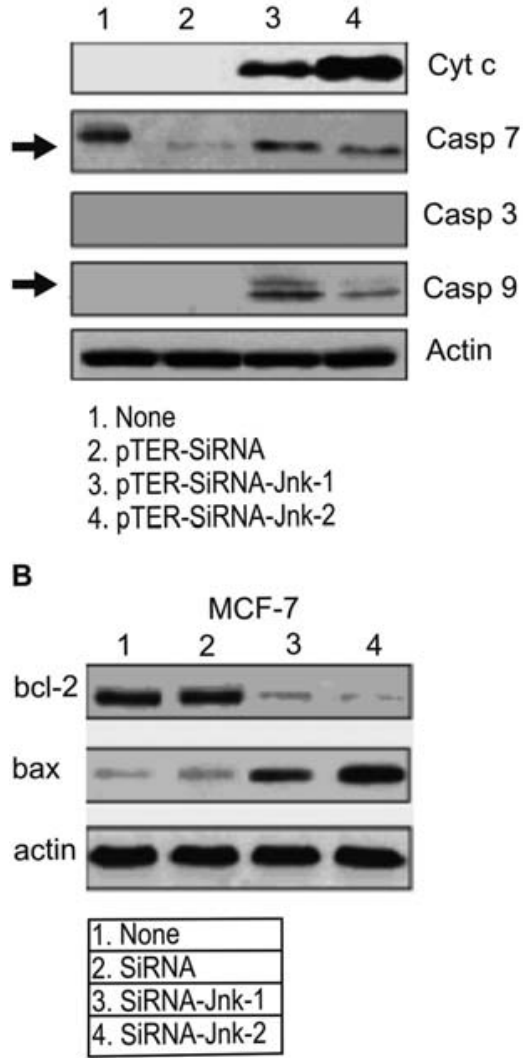

Figure 6. Caspase activity and apoptotic related markers. (A) Western blot analysis of caspases in MCF-7 cells transfected with 1, none; 2, pTERsiRNA; 3, pTER-siRNA-Jnk-1 and 4, pTER-siRNA-Jnk-2. (B) Protein levels of Bcl-2, and Bax following different treatment. Protein $(50 \mu \mathrm{g})$ was loaded for the controls and treated samples: 1, none; 2, pTER-siRNA; 3, pTER-siRNA-Jnk-1 and 4, pTER-siRNA-Jnk-2. One of two similar experiments is shown.

chondrial apoptotic pathways in a variety of cell systems (18). Therefore, we examined the possible involvement of JNK pathway in the induced apoptosis in MCF-7 cells. JNK activation was determined by Western blot analysis after 48-h treatment of cells with two siRNA against Jnk-1 and Jnk-2 mRNAs. Our data indicated that both, siRNA-Jnk-1 and siRNA-Jnk-2 induce antiproliferative effects in a timedependent manner. We demonstrated that siRNA-Jnk-1 and siRNA-Jnk-2 induced apoptosis in MCF-7 cells by downregulation of bcl-2, and up-regulation of bax, which led to the release of cytochrome $\mathrm{c}$ from the mitochondria into the cytosol, which, in turn, resulted in the activation of caspase- 9 and caspase-7, and ultimately in cell death. The induction of the mitochondrial caspase-dependent pathway was confirmed by pretreatment with pan-caspase inhibitor z-VAD-fmk and antioxidant $\mathrm{N}$-acetyl-L-cysteine. As expected MCF-7 cells did not exhibit any caspase-3 activity (Fig. 6A).

Apoptosis related markers. Effect of siRNA-Jnk-1 and siRNA-Jnk-2 on Bcl-2 and Bax. To investigate whether pTER, pTER-siRNA-Jnk-1 or pTER-siRNA-Jnk-2 -induced apoptosis in MCF-7 cells correlates with expression of proteins known to be involved in the apoptotic process, we performed Western blot analysis of Bcl-2 and Bax in MCF-7 
cells treated with pTER-siRNA-Jnk-1 or siRNA-Jnk-2 for 48 h. As shown in Fig. 6B, both, siRNA-Jnk-1 and siRNAJnk-2 reduced $\mathrm{Bcl}-2$ protein concentration in $\mathrm{MCF}-7$ cells, but increased Bax protein concentration in MCF-7 cells.

\section{Discussion}

Cancer cells often show alteration in the signal-transduction pathways, leading to proliferation in response to external signals. The kinases Jnk-1 and Jnk-2 are expressed in a high proportion of most human cancers, including breast, prostate, gastrointestinal cancer, lymphoma, melanoma, and myeloid leukemia (21-25). Altered expression of JNK-1 or JNK-2 seems to define a common event associated with the pathogenesis of several human cancers (26-28). Previous studies in prostate cancer demonstrated that the continued presence of JNK $1 / 2$ were required for cancer development in combination with cisplatin (9) and that inactivation of Jnk-1/2 resulted in the sustained regression of tumors $(9,29)$. Several observations suggest that the JNK/SAPK pathway may play a major role in transformation process (30-32). Similarly, activation of JNK pathway is essential for transformation of Fisher rat fibroblasts by the MET/Hepatocyte Growth Factor receptor pathway (33). Other studies indicate that some growth factors including EGF may cause the activation of the JNK pathway mediated by the activation of phosphoinositol3-kinase (24,34-36). Our results demonstrated that siRNA can effectively down-regulate Jnk-1 or Jnk-2 overexpression with great specificity. We showed that the plasmids endogenously expressing siRNA could successfully deplete up to $80 \%$ of JNK-1 and JNK-2 expression in MCF-7 cells at day 2 after transfection. Furthermore, the inhibition effects persisted for at least 8 days after transfection in dishes as shown by experiments in vitro, even though the protein level of JNK $1 / 2$ in silenced clones expressing siRNA was back to almost the same level as in the control cells by day 8 after transfection. Although some studies previously revealed that the effects of inactivation of Jnk-1/2 in some cell lines were modest (37), other groups using different approaches to reduce the protein level of JNK-1/2 found that a decrease in JNK-1/2 expression could inhibit the growth of several tumor cells, including breast tumor cells (37-39). In several breast carcinoma cell lines where EGF is known to have a growth inducing effect, such as MCF-7 cells, mitogenesis is accompanied by induction of Fos and Jun family members $(40,41)$. $\mathrm{EGF}$ is mitogenic for MCF-7 and a potent activator of AP-1 activity (41). These results are consistent with a causal role of JNK/SAPK in transformation of MCF-7 breast carcinoma. It has been reported that mJun inhibits DNA repair and, when stably expressed in MCF-7 cells, inhibits transformation including tumorigenesis and sensitizes the cells to cisplatin (42). There have previously been conflicting reports about the role of Jnk-1/2 in apoptosis (43). Constant overexpression of JNK-1/2 might induce apoptosis $(22,43,44)$, and a decrease in JNK-1/2 levels by techniques brought about by, for example, an antisense approach might also cause apoptosis of certain tumor cells (37) or might increase the sensitivity of the cells to apoptotic stimuli (9). These conflicting observations suggested that Jnk was capable of both inducing and suppressing apoptosis in different types of tumor cell, under different conditions and in different systems. In prostate carcinoma cell lines PC-3 and LNCaP and in T98G glioblastoma cells, both JNK1 and JNK-2 isoforms participate in promoting proliferation to high density. However, the pathways that Jnk-1/2 controls and/or that are involved in the observed apoptosis remain obscure. Several reports showed that JNK phosphorylates the anti-apoptotic protein Bcl-2 and $\mathrm{Bcl}-\mathrm{x}$, although the consequences of this phosphorylation are not clear. It is possible that phosphorylation of these proteins impair their anti-apoptotic function, contributing to the induction of apoptosis (45). Understanding the precise pathway by which a decrease in JNK-1/2 in MCF-7 cells by siRNA was able to induce apoptosis upon serum deprivation needs further study. Inactivation of Egr-1 by RNA interference in prostate cancer cells resulted in induction of apoptosis and lost of the anchorage capacity $(14,15)$. In addition, we measured caspase- 3 activity in MCF-7 cells grown in $10 \%$ FCS treated or not with siRNA against Jnk-1 or Jnk-2. As expected MCF-7 cells did not exhibit any caspase- 3 activity. Caspase- 3 activity is thought to be essential for DNA fragmentation. With regard to the caspase cascade, caspase-9 was activated in the MCF-7 cell line after transfected with siRNA against Jnk-1 or Jnk-2 and further increased at the end of the experiment. Similar behavior was observed for caspase-7. Whereas caspase-6 cleavage (data not shown) was not induced by any of the treatments. The sum of the results suggests that the phosphorylation of the anti-apoptotic gene and persistent up-regulation of proapoptotic markers were induced by siRNA-Jnk-1 or siRNAJnk-2 in MCF-7 cells. These results indicate that the mitochondrial instability led to apoptosis through the cytochromec-mediated activation of caspase -9 and -7 . These data are consistent with the suggestion that the siRNA against Jnk-1 or Jnk-2 is capable of inducing DNA fragmentation and apoptosis in MCF-7 cells, suggesting that inhibition of Jnk-1 or Jnk-2 activity by siRNA may utilize an alternative caspase pathway to induce apoptosis. In summary, we show that siRNA against Jnk-1 and Jnk-2 promote apoptosis in the MCF-7 human breast cancer cell line, and this effect is partly due to down-regulation of $\mathrm{Bcl}-2$ protein.

\section{Acknowledgements}

We thank Dr Anna Bigas Salvans (Laboratory of Transcriptional Control of Cell Differentiation, Institut Recerca Oncologica, Barcelona, Spain) for providing the pTER vector for expressing the siRNA-Jnk-1 and Jnk-2. This study was supported by the Chilean National Science Foundation FONDECYT regular grant award 1060774.

\section{References}

1. Jenal A, Thomas A, Murry $\mathrm{T}$ and Thun M: Cancer stastics. CA Cancer J Clin 52: 23-37, 2002.

2. Chan K and Morris GJ: Chemoprevention of breast cancer for women at high risk. Semin Oncol 33: 642-646, 2006.

3. Shimada K, Nakamura M, Ishida E, Kishi M and Konishi N: Roles of p38- and c-jun NH2-terminal kinase-mediated pathways in 2-methoxyestradiol-induced p53 induction and apoptosis. Carcinogenesis 24: 1067-1075, 2003.

4. Fan Y, Chen H, Qiao B, et al: Opposing effects of ERK and p38 MAP kinases on HeLa cell apoptosis induced by dipyrithione. Mol Cells 23: 30-38, 2007. 
5. Davis RJ: Signal transduction by the JNK group of MAP kinases. Cell 103: 239-252, 2000.

6. Tournier C, Hess P, Yang D, Xu J, Turner TK, Nimnual A, Bar-Sagi D, Jones SN, Flavell RA and Davis RJ: Requirement of JNK for stress-induced activation of the cytochrome c-mediated death pathway. Science 288: 870-874, 2000.

7. Bivik CA, Larsson PK, Kågedal KM, Rosdahl IK and Öllinger KM: UVA/B-induced apoptosis in human melanocytes involves translocation of cathepsins and Bcl-2 family members. J Invest Dermatol 126: 1119-1127, 2006.

8. Dietrich N, Thastrup J, Holmberg C, Gyrd-Hansen M, Fehrenbacher N, Lademann U, Lerdrup M, Herdegen T, Jäättelä M and Kallunki T: JNK2 mediates TNF-induced cell death in mouse embryonic fibroblasts via regulation of both caspase and cathepsin protease pathways. Cell Death Differ 11: 301-313, 2004

9. Potapova O, Haghighi A, Bost F, Liu C, Birrer MJ, Gjerset R and Mercola D: The Jun kinase/stress-activated protein kinase pathway functions to regulate DNA repair and inhibition of the pathway sensitizes tumor cells to cisplatin. J Biol Chem 272: 14041-14044, 1997.

10. Konnikova L, Kotecki M, Kruger MM, Cochran BH and Miao GY: Downregulation of survivin gene expression by RNAi knockdown of STAT3 expression by RNAi induces apoptosis in astrocytoma cells. BMC Cancer 3: 23, 2003.

11. Sioud M, Furset G and Cekaite L: Suppression of immunostimulatory siRNA-driven innate immune activation by 2'modified RNAs. Biochem Biophys Res Commun 361: 122-126, 2007.

12. Stevenson M: Therapeutic potential of RNA interference. Engl J Med 351: 1772-1777, 2004.

13. Devroe E and Silver PA: Retrovirus-delivered siRNA. BMC Biotechnol 2: 15-20, 2002 .

14. Parra E, Ortega A and Saenz L: Downregulation of Egr-1 by siRNA inhibits growth of human prostate carcinoma cell line PC-3. Oncol Rep 22: 1513-1518, 2009.

15. Parra E and Ferreira J: The effect of siRNA-Egr-1 and camptothecin on growth and chemosensitivity of breast cancer cell lines. Oncol Rep 22: 1159-1165, 2010

16. Persengiev SP, Zhu X and Green MR: Non-specific, concentration-dependent stimulation and repression of mammalian gene expression by small interfering RNAs (siRNA). RNA 10: $12-18,2004$.

17. Sui G, Soohoo C, Affar E, Gay F, Shi Y, Forrester WC and Shi Y: A DNA vector based RNAi technology to suppress gene expression in mammalian cells. Proc Natl Acad Sci USA 99: 5515-5520, 2002

18. Bossy-Wetzel E, Bakiri L and Yaniv M: Induction of apoptosis by the transcription factor c-Jun. EMBO J 16: 1695-1709, 1997.

19. Collins S, Lutz MA, Zank PA, Anders R, Kersh G and Powell JD: Opposing regulation of T cell function by Egr-1/ NAB2 and Egr-2/Egr-3. Eur J Immunol 38: 528-536, 2008

20. Amorino G, Hamilton V, Valerie K, Dent P, Lammering G and Schmidt-Ullrich R: Epidermal growth factor receptor dependence of radiation-induced transcription factor activation in human breast carcinoma cells. Mol Biol Cell 13: 2233-2244, 2002.

21. Inostroza J, Saenz L, Calaf G, Cabello G and Parra E: Role of the phosphatase PP4 in the activation of JNK-1 in prostate carcinoma cell lines PC-3 and LNCaP resulting in increased AP-1 and EGR-1 activity. Biol Res 38: 163-178, 2005

22. Xiao L and Lang WA: A dominant role for the c-Jun NH2terminal kinase in oncogenic ras-induced morphologic transformation of human lung carcinoma cells. Cancer Res 60 : 400-408, 2000.

23. Risse-Hackl G, Adamkiewicz J, Wimmel A and Schuermann M: Transition from SCLC to NSVLC phenotype is accompanied by an increased TRE-binding acticity and recruitment of specific AP-1 proteins. Oncogene 16: 3057-3068, 1998.

24. Bost F, McKay R, Dean N and Mercola D: The JUN kinase/ stress-activated protein kinase pathway is required for epidermal growth factor stimulation of growth of human A549 lung carcinoma cells. J Biol Chem 272: 33422-33429, 1997.

25. Potapova O, Gorospe M, Bost F, Dean NM, Gaarde W, Mercola D and Holbrook N: c-Jun N-terminal kinase is essential for growth of human T98G glioblastoma cells. J Biol Chem 275: 24767-24775, 2000 .
26. Crul M, Schellens JHM, Beijnen JH and Maliepaard M: Cisplatin resistance and DNA repair. Cancer Treat Rev 23: 341-366, 1997.

27. Delmastro DA, Li V, Vaisman A, Solle M and Charney SG: DNA damage inducible-gene expression following platinum treatment in human ovarian carcinoma cell lines. Cancer Chemother Pharmacol 39: 245-253, 1997.

28. Jordan P and Carmo-Fonseca M: Molecular mechanisms involved in cisplatin cytotoxicity. Cell Mol Life Sci 57: $1229-1235,2000$

29. Persons DL, Yazlovitskaya EM, Cul W and Pelling JC: Cisplatin-induced activation of mitogen-activated protein kinases in ovarian carcinoma cells: inhibition of extracellular signal-regulated kinase activity increases sensitivity to cisplatin. Clin Cancer Res 5: 1007-1014, 1999.

30. Potapova O, Basu S, Mercola D and Holbrook NJ: Protective role for c-Jun in the cellular response to DNA damage. J Biol Chem 276: 28546-28553, 2001.

31. Smeal T, Binetruy B, Mercola D, Birrer M and Karin M: Oncogenic and transcriptional cooperation with Ha-Ras requires phosphoryaltion of c-Jun on serines 63 and 73. Nature 354: 494-496, 1991.

32. Song HY, Rognier CH, Kirschning CJ, Goeddel DV and Rothe M: Tumor necrosis factor (TNF) mediated kinase cascades: bifurcation of nuclear factor kappa $\mathrm{B}$ and $\mathrm{c}$ jun $\mathrm{N}$ terminal kinase (JNK/SAPK) pathways at TNF receptor associated factor 2. Proc Natl Acad Sci USA 94: 9792-9796, 1997.

33. Rodrigues GA, Park M and Schlissinger J: Activation of the JNK pathway is essential for transformation by the Met oncogene. EMBO J 16: 2634-2645, 1997.

34. Antonyak MA, Moscatello D and Wong A: Constitutive activation of c-Jun N-terminal kinase by a mutant epidermal growth factor receptor. J Biol Chem 273: 2817-2822, 1998.

35. Logan S, Falasca M, Hu P and Schlessinger J: Phosphatidylinositol 3-kinase mediates epidermal growth factor-induced activation of the c-Jun $\mathrm{N}$-terminal kinase signaling pathway. Mol Cell Biol 7: 5784-5789, 1997.

36. Minden A, Lin A, Claret FX, Abo A and Karin M: Selective activation of the JNK signaling cascade and c-Jun transcriptional activity by the small GTPases Rac and Cdc $42 \mathrm{Hs}$. Cell 81: 1147-1157, 1995.

37. Hayakawa J, Ohmichi M, Kurach H, Ikegami H, Kimura A, Matsuoka T, Jikihara H, Mercola D and Murata Y: Inhibition of extracellular signal-regulated protein kinase or c-Jun N-terminal protein kinase cascade, differentially activated by cisplatin, sensitizes human ovarian cancer cell-line. J Biol Chem 274: 31648-31654, 1999.

38. Yong-Min Y, Bost F, Charbono W, Dean N, McKay R, Depatie C and Mercola D: C-Jun NH2-terminal kinase mediates proliferation and tumor growth in human prostrate carcinoma. Clin Cancer Res 9: 391-401, 2003.

39. Hayakawa J, Depatie C, Ohmichi M and Mercola D: The stress response of Jun kinase to DNA-damaging drugs serves to promote drug resistance via phospho-ATF2-dependent DNA repair. J Biol Chem 278: 20582-20592, 2003.

40. Banerjee S, Sengupta K, Saxena NK, Dhar K and Banerjee SK: Epidermal growth factor induces WISP-2/CCN5 expression in estrogen receptor-alpha-positive breast tumor cells through multiple molecular cross-talks. Mol Cancer Res 3: 151-159, 2005.

41. Chen TK, Smith LM, Gebhardt DK, Birrer M and Brown PH: Activation and inhibition of the AP- 1 complex in human breast cancer cells. Mol Carcinog 15: 215-226, 1996.

42. Hayakawa J, Depatie C, Ohmichi M and Mercola D: The activation of c-Jun NH2-terminal kinase (JNK) by DNAdamaging agents serves to promote drug resistance via activating transcription factor 2 (ATF2)-dependent enhanced DNA repair. J Biol Chem 278: 20582-20592, 2003.

43. Adler V, Fuchs S, Kim J, Kraft A, King MP, Pelling J and Ronai Z: jun-NH2-terminal kinase activation mediated by UVinduced DNA lesions in melanoma and fibroblast cells. Cell Growth Differ 6: 1437-1446, 1995.

44. Monno S, Newman MV, Cook M and Lowe WL: Insulin-like growth factor I activates c-Jun N-terminal kinase in MCF-7 breast cancer cells. Endocrinology 141: 544-550, 2000.

45. Yamamoto K, Ichijo $\mathrm{H}$ and Korsmeyer SJ: BCL-2 is phosphorylated and inactivated by an ASK1/Jun N-terminal protein kinase pathway normally activated at $\mathrm{G}(2) / \mathrm{M}$. Mol Cell Biol 19: 8469-8478, 1999 